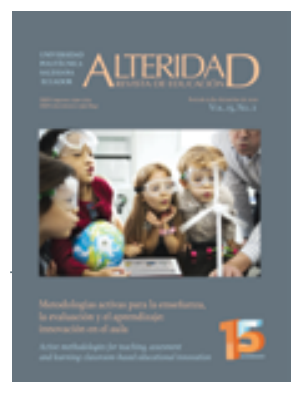

\title{
El rendimiento académico y la evaluación formativa y compartida en formación del profesorado
}

\section{Academic performance and formative and shared assessment in teacher education}

Diriam Molina-Soria es docente e investigadora de la Universidad de Valladolid (España) (miriam.molina@uva.es) (http://orcid.org/0000-0003-2974-5535)

(D) Cristina Pascual-Arias es docente e investigadora de la Universidad de Valladolid (España) (cristina.pascual@uva.es) (http://orcid.org/0000-0002-2781-5600)

(D) Dr. Víctor Manuel López-Pastor es docente e investigador de la Universidad de Valladolid (España) (vlopez@mpc.uva.es) ( http://orcid.org/0000-0003-2681-9543)

Recibido: 2020-01-31 / Revisado: 2020-06-06 / Aceptado: 2020-06-08 / Publicado: 2020-07-01

\section{Resumen}

En la última década se está despertando un elevado interés por las ventajas que la aplicación de una evaluación formativa parece tener en la docencia universitaria. El presente estudio tiene como finalidad analizar si la Evaluación Formativa y Compartida en la Formación Inicial del Profesorado ayuda al alumnado a obtener mejores resultados académicos. Para ello se realiza un estudio de caso con 37 alumnos de la asignatura de Expresión y Comunicación Corporal en la Educación Infantil de cuarto curso del Grado de Educación Infantil. El alumnado puede elegir entre tres vías de aprendizaje y evaluación: Vía continua, vía mixta y vía final o no presencial. Tras el análisis de las actas oficiales de calificación, los resultados obtenidos muestran que la Evaluación Formativa y Compartida ha influido en su rendimiento académico, y el alumnado que ha optado por la vía de evaluación continua ha obtenido un mejor rendimiento académico. La mayoría del alumnado ha optado por esta vía de aprendizaje y evaluación. El 97.3\% del alumnado ha superado la asignatura y la media de las calificaciones es de notable. Por esta razón, parece necesario investigar más a fondo el tema con muestras más grandes y, especialmente, ante la nueva situación de confinamiento y enseñanza on-line.

Descriptores: Evaluación formativa, evaluación compartida, rendimiento académico, formación inicial del profesorado, educación física, educación infantil.

\section{Abstract}

In the last decade a high interest is being aroused by the advantages that the application of a formative assessment seems to have in university teaching. The purpose of this study is to analyze whether the Formative and Shared Assessment in Pre-service Teacher Education helps students obtain better academic results. It's a case study is carried out with 37 students of the subject of Expression and Body Communication in Early Childhood Education in the fourth year of the Early Childhood Education Degree. Students can choose between three ways of learning and assessment: Continuous track, mixed track and final or nonface route. After the analysis of the official records of mark, the results obtained show that the Formative and Shared Assessment has influenced their academic performance, and the students who have opted for the continuous assessment pathway have obtained a better academic performance. Most students have opted for this way of learning and assessment. 97.3\% of students have passed the subject and the average of the students grades is remarkable. For this reason, it seems necessary to investigate further the topic with larger samples and, especially, in the face of the new situation of confinement and online teaching.

Keywords: Formative assessment, shared assessment, academic performance, pre-service teacher education, physical education, early childhood education.

Forma sugerida de citar: Molina-Soria, M., Pascual-Arias, C., \& López-Pastor, V. (2020). El rendimiento académico y la evaluación formativa y compartida en formación del profesorado. Alteridad, 15(2), $204-215$. https://doi.org/10.17163/alt.v15n2.2020.05 


\section{Introducción y estado de la cuestión}

Desde hace años, el profesorado universitario que se encarga de la Formación Inicial del Profesorado (FIP) está buscando un cambio con respecto a la evaluación. Buscan pasar de la "cultura del examen" o "aprendizaje bancario" a la "cultura de la evaluación" o "aprendizaje dialógico" (Dochy et al., 2002). La "cultura del examen" es una metodología tradicional, basada en las lecciones magistrales en las que el conocimiento está centrado en el profesor y se evalúan únicamente los resultados finales, mientras que la "cultura de la evaluación" tiene como finalidad generar procesos de evaluación aportando retroalimentación para generar un mayor aprendizaje en los alumnos y mejorar el proceso de enseñanza-aprendizaje; de esta forma, no se da tanta importancia a la calificación (López-Pastor et al., 2020). Por ello, muchos autores consideran que para mejorar el proceso de enseñanza-aprendizaje, es necesario llevar a cabo procesos de Evaluación Formativa y Compartida (EFyC) (Cañadas et al., 2018; Carter-Thuillier, 2015; Gallardo et al., 2019a; Gallardo et al., 2019b; López-Pastor et al., 2020; Romero-Martín et al., 2016), con un especial atención al feedback y la participación del alumnado en el aprendizaje (Biggs, 2005; Black \& William, 2003; Boud, 2010; Boud \& Falchikov, 2006; Brown \& Glasner, 2003; Falchikov, 2005; Nicol \& Macfarlane-Dick, 2006).
Según Castejón et al. (2011), la evaluación formativa se centra en mejorar los procesos de enseñanza aprendizaje. En este sentido, LópezPastor et al. (2020) afirman que la evaluación formativa busca generar procesos de mejora y aprendizaje en tres sentidos: (a) Mejorar los procesos de aprendizaje del alumnado y la calidad de sus producciones; (b) ir mejorando poco a poco la práctica docente; $y$, (c) reconducir los procesos de enseñanza-aprendizaje que se llevan a cabo en el aula, a lo largo de la asignatura y curso a curso.

López-Pastor y Pérez-Pueyo (2017) definen la evaluación compartida como los diálogos, individuales o grupales que se realizan entre el profesorado y el alumnado, sobre los procesos de enseñanza-aprendizaje realizados previamente. La evaluación compartida favorece la participación del alumnado en el proceso de evaluación y ha demostrado que también mejora el proceso de aprendizaje de los alumnos (Gallardo et al., 2019a; Carter-Thuillier, 2015; Moreno et al., 2019; López-Pastor \& Pérez-Pueyo, 2017).

Actualmente, diversos estudios defienden la importancia de la participación del alumnado en los procesos de evaluación (Boud, 2010; Boud \& Falchikov, 2006; Brown \& Glasner, 2003; Falchikov, 2005; Ibarra et al., 2012; Herranz, 2013). Además, López-Pastor y Pérez-Pueyo (2017) defienden la par ticipación del alumnado en el proceso de evaluación a través de diferentes técnicas, que se pueden resumir en la siguiente tabla (ver tabla 1):

Tabla 1. Técnicas de participación del alumnado en evaluación (a partir de López-Pastor \& Pérez-Pueyo, 2017)

\begin{tabular}{|c|l|}
\hline Autoevaluación & $\begin{array}{l}\text { Es la evaluación que se realiza una persona a sí misma. Puede evaluar su proceso y/o el resultado } \\
\text { personal que ha obtenido. Esta técnica puede realizarse con carácter individual o grupal. }\end{array}$ \\
\hline Coevaluación & Es la evaluación entre iguales (de manera individual o grupal). \\
\hline $\begin{array}{c}\text { Evaluación } \\
\text { compartida }\end{array}$ & $\begin{array}{l}\text { Procesos de diálogo que se realizan entre el profesorado y el alumnado, sobre los procesos de ense- } \\
\text { ñanza-aprendizaje realizados previamente. Pueden ser individuales, en pequeños grupos o colectivos. }\end{array}$ \\
\hline Autocalificación & $\begin{array}{l}\text { El alumno fija una calificación que piensa que merece. Es importante establecer previamente unos } \\
\text { criterios de calificación por parte del profesorado. Estos criterios deben ser públicos desde el inicio de } \\
\text { la asignatura y es adecuado consensuarlos con los estudiantes. }\end{array}$ \\
\hline $\begin{array}{c}\text { Calificación } \\
\text { dialogada }\end{array}$ & $\begin{array}{l}\text { Es el proceso que se realiza entre el profesorado y el alumnado para dialogar sobre la calificación } \\
\text { definitiva. Para ello, también es importante que los criterios de calificación estén previamente estable- } \\
\text { cidos. Este proceso se puede dar de manera individual, en pequeños grupos o en gran grupo. }\end{array}$ \\
\hline
\end{tabular}

Fuente: A partir de López-Pastor y Pérez-Pueyo (2017). 
Pueden encontrarse algunos estudios sobre el desarrollo de experiencias de EFyC en Latinoamérica. Gallardo et al (2017) revisan la percepción de alumnado, profesores y egresados de una universidad chilena sobre la posible aplicación de este tipo de sistemas durante la FIP. Posteriormente, investigan en qué medida la aplicación de sistemas de EFyC influye en la autopercepción de competencias adquiridas en FIP (Gallardo et al, 2018), así como las ventajas e inconvenientes de aplicar este tipo de evaluación en la FIP en universidades chilenas (Gallardo et al, 2020). En la misma línea, Moreno et al. (2019) realizan un estudio discursivo sobre la evaluación formativa y la participación del alumnado en una actividad de feedback en una universidad pública de México. Se realizan tres categorías de análisis de datos: La coevaluación, la evaluación entre pares y la autoevaluación. Los resultados muestran que los alumnos que reciben el feedback participan en su evaluación cuestionando y añadiendo datos relevantes para que se produzca un mejor aprendizaje.

Existen estudios que indican que gracias al implemento de los sistemas de EFyC, el alumnado mejora su rendimiento académico (Angelini, 2016; Arribas, 2012; Gallardo et al., 2020; López-Pastor, 2008; Castejón et al., 2011; Fraile et al., 2013; Romero-Martín et al., 2014). Entendemos que el rendimiento académico son las calificaciones que obtienen los alumnos al finalizar la asignatura.

López-Pastor (2008) realiza un estudio de caso en la FIP de educación física en la que encuentra un alto rendimiento académico del alumnado. Además, en dicho estudio encontramos una serie de razones por las que es beneficioso poner en práctica sistemas de EFyC en la FIP: (a) porque permite mejorar los procesos de enseñanza-aprendizaje y como consecuencia, aumenta su motivación e implicación en dichos procesos; (b) porque es la evaluación más coherente si se utilizan metodologías activas y sistemas centrados en el aprendizaje del alumnado; y (c) porque estos sistemas desarrollan la responsabilidad, la autonomía y la autocrítica en los procesos de aprendizaje.
Castejón et al. (2011) realizan un estudio sobre la utilización de los sistemas de EFyC en la FIP de educación física para la mejora el rendimiento académico del alumnado de tres universidades españolas a través de un análisis estadístico descriptivo. El alumnado tiene la opción de elegir la vía de aprendizaje según el tipo de evaluación: continua o final. La elevada asistencia y la participación del alumnado en los procesos de evaluación son muy importantes para poder realizar la vía de evaluación continua. Estos autores llegan a la conclusión de que la utilización de sistemas de EFyC puede ayudar a lograr un mejor rendimiento académico del alumnado que opta por la vía continua frente a los alumnos que eligen la vía final. Por otra parte, Fraile et al. (2013) desarrollan un estudio para analizar la influencia de la aplicación de sistemas de EFyC en la FIP en el rendimiento académico. Se realiza un análisis estadístico descriptivo y ANOVAS sobre los datos de rendimiento académico de 19 universidades españolas en 52 asignaturas diferentes. Se ofrece al alumnado varias vías de evaluación y se comparan la EFyC vs vía mixta o examen final. Los porcentajes del alumnado que trabaja en la asignatura desarrollando sistemas de EFyC son un $83 \%$ de aptos (en su mayoría, notables), un $8 \%$ no presentados y un $9 \%$ suspensos. Por ello, los resultados obtenidos parecen indicar que el alumnado que desarrolla sistemas de EFyC alcanza mejores calificaciones que el alumnado que opta por la vía final o examen final. Además, en universidades anglosajonas, Boud y Falchikov (2006) realizan estudios sobre la fiabilidad que tienen los procesos de participación el alumnado en la evaluación, con resultados positivos. Boud (2010) afirma que es bueno involucrar a los alumnos en su proceso de evaluación porque mejora activamente su aprendizaje.

Por su parte, Arribas (2012) realiza un estudio sobre el rendimiento académico según el sistema de evaluación utilizado. Utiliza una muestra de 2192 alumnos de catorce universidades españolas. Los resultados indican que el sistema de evaluación utilizado tiene influencia 
sobre el rendimiento académico del alumnado $y$, que la evaluación continua es la que mejores resultados académicos genera. De igual forma, Romero-Martín et al. (2014) analizan la influencia que tienen los sistemas de EFyC en la FIP de quince universidades españolas, con una muestra de 3625 alumnos de 30 asignaturas diferentes a través de un análisis estadístico descriptivo y ANOVAS. Los resultados presentados indican que el alumnado que experimenta sistemas de EFyC mejora su rendimiento académico y está satisfecho con este tipo de evaluación porque participa y mejora su aprendizaje. Otro estudio de Romero-Martín et al. (2015), en el que analizan las divergencias del profesorado y del alumnado en la FIP tras aplicar sistemas de EFyC, obtienen como resultados que, aunque la $\mathrm{EFyC}$ es muy exigente para el alumnado, se muestra muy satisfecho con la calificación final de la asignatura.

Gallardo et al. (2020) realizan un estudio en la universidad chilena sobre la percepción de los alumnos de FIP de educación física y de formación profesional Técnico Deportivo Universitario sobre el sistema de EFyC utilizado en asignaturas de atención a la diversidad. Los resultados muestran que los alumnos valoran positivamente el sistema de EFyC que se ha llevado a cabo durante las asignaturas, a pesar de que consideren que exige una asistencia obligatoria, continuidad y mayor tiempo y esfuerzo. Estos inconvenientes sobre el sistema de evaluación utilizado consideran que se ven recompensados con un mayor aprendizaje y un mejor rendimiento académico.

López et al. (2016) afirman que la utilización de sistemas de EFyC está relacionada con la utilización de metodologías activas de aprendizaje. En este sentido, López-Pastor et al. (2020) resaltan la importancia de utilizar metodologías activas y sistemas de EFyC como alternativa a las metodologías tradicionales, porque que es la evaluación más lógica. Según Castejón et al. (2011), parece que existe un vínculo entre la utilización de metodologías activas y los sistemas de EFyC, ya que el profesor y el alumno trabajan de manera colaborativa a través de una retroalimentación constante para mejorar el proceso de enseñanza-aprendizaje: Orienta la toma de decisiones del profesorado y del alumnado, regula las actuaciones docentes, se pueden establecer ciclos de reflexión-acción, etc.

Por todo ello, el objetivo principal de esta investigación, es analizar si los sistemas de EFyC en la FIP ayudan al alumnado a obtener mejores resultados académicos por la vía continua de aprendizaje que si sigue la vía final o no presencial.

\section{Material y métodos}

El diseño de esta investigación es un estudio de caso simple, ya que se analiza un caso único, de un solo grupo de alumnos. Según Martínez (2006), con el método de estudio de caso se mide y registra la conducta de las personas del fenómeno que queremos estudiar a través de una rigurosidad científica que demuestre validez y fiabilidad en los resultados. Un estudio de caso se caracteriza por ser un estudio descriptivo que tiene una única muestra, ya sea una persona o un grupo de personas (Montero \& León, 2005). En esta investigación se va a analizar un caso concreto de una única asignatura, en un contexto real de la FIP.

El contexto en el que se lleva a cabo este estudio es una Facultad de FIP de Segovia (Universidad de Valladolid, España), concretamente en la asignatura de Expresión y Comunicación Corporal en la Educación Infantil que se desarrolla en el primer cuatrimestre del curso 2019-2020. Es una asignatura de 6 créditos ECTS (150 horas) de carácter optativo. La muestra está compuesta por 37 alumnas y alumnos de cuarto curso del Grado de Educación Infantil y de quinto curso del Programa de Estudios Conjunto de Grado en Educación Infantil y Grado en Educación Primaria. Los grados de maestro en España tienen una duración de cuatro años y equivalen a 240 créditos ECTS. La Facultad de Educación de Segovia oferta dos tipos: Grado 
en Educación Infantil y Grado en Educación Primaria. Y, además, se oferta un Programa de Estudios Conjunto de Grado en Educación Infantil y Grado en Educación Primaria con una duración de 5 años. En el último curso del grado se debe elegir la mención a desarrollar compuesta por cinco asignaturas especializadas en la temática elegida. Las menciones en el Grado de Educación Infantil en este centro son: (a) Expresión y Comunicación Artística y Motricidad; y (b) Observación y exploración del entorno. Las menciones para el Grado de Educación Primaria son: (a) Educación Musical; (b) Educación Física; y (c) Entorno, Naturaleza y Sociedad.

La asignatura se desarrolla durante trece semanas programadas del mes de septiembre al mes de diciembre. Se imparten dos horas de práctica, una hora de teoría y una hora de seminario a la semana. En la tabla 2, se desarrolla una previsión de la dedicación del alumnado a la asignatura:

Tabla 2. Horas de dedicación del alumnado a la asignatura

\begin{tabular}{|l|c|l|c|}
\hline \multicolumn{1}{|c|}{ Actividades presenciales } & Horas & \multicolumn{1}{|c|}{ Actividades no presenciales } & Horas \\
\hline Clases teórico-prácticas & 30 & Estudio y trabajo autónomo individual & 40 \\
\hline Clases prácticas de aula & 23 & Estudio y trabajo autónomo grupal & 40 \\
\hline Seminarios & 7 & & 5 \\
\hline Tutorías grupales & & & 5 \\
\hline Evaluación & & & 90 \\
\hline Total presencial & 60 & Total no presencial & 5 \\
\hline
\end{tabular}

\section{Elaboración propia.}

El estudio se centra en uno de los resultados que genera el sistema de evaluación utilizado, así que vamos a explicarlo con más detalle en este apartado del contexto. Cuando el primer día de clase se presenta y explica la organización de la asignatura, se le ofrecen al alumnado tres vías de aprendizaje y evaluación: (a) vía continua, (b) vía mixta y (c) vía final o no presencial (ver tabla 3). El alumnado puede elegir la vía que más se adapte a su situación personal, aunque en ocasiones y según transcurre la asignatura, hay personas que pasan de vía continua a vía mixta. Según Castejón et al. (2011) y Biggs (2005), el alumnado realiza la elección del sistema de aprendizaje y evaluación en el contexto de las metodologías activas y de los sistemas de EFyC.

En la Tabla 3 presentamos los requisitos que debe cumplir el alumnado para cada vía de evaluación, así como los porcentajes sobre la calificación final otorgados a cada actividad de aprendizaje. Es importante señalar que, el primer día de clase, se dialoga con el alumnado para ver si está de acuerdo en el peso de cada actividad sobre la calificación, o si quiere establecer alguna modificación.

Los alumnos tienen escalas descriptivas con los criterios de evaluación y calificación para cada actividad de aprendizaje. Es importante señalar que, para poder aprobar, hay que superar cada apartado. Todos los trabajos y actividades de aprendizaje entregados se devuelven corregidos por el profesor en el plazo de una semana, y gracias al feedback aportado, los alumnos pueden mejorar el trabajo en el mismo plazo de tiempo.

En la vía continua, el alumnado sigue un proceso continuo y formativo, con un seguimiento y feedback constante, sin necesidad de realizar un examen final. En la vía mixta, están los alumnos que no pueden asistir al 100\% de las clases de la asignatura, pero sí realizan trabajos y siguen el funcionamiento habitual de las clases. La vía final o no presencial, es para el alumnado que no asiste a ninguna clase, no ha realizado ningún trabajo ni actividad de aprendizaje; se basa en una evaluación final y sumativa. 
Tabla 3. Requisitos para las distintas vías de aprendizaje y evaluación y peso en la calificación final de la asignatura

\begin{tabular}{|c|c|c|}
\hline Vía continua & Vía mixta & Vía no presencial \\
\hline $\begin{array}{l}\text { Asistencia a todas las clases (solo se pue- } \\
\text { de faltar un } 15 \% \text { de las clases de manera } \\
\text { justificada) } \\
\text { Requiere la entrega de todos los trabajos } \\
\text { de la asignatura } \\
\text { Existe un examen parcial con coevaluación }\end{array}$ & $\begin{array}{l}\text { Asistencia, al menos, al } 50 \% \text { de las } \\
\text { clases } \\
\text { No es obligatorio entregar los trabajos } \\
\text { Existe un examen parcial con } \\
\text { coevaluación }\end{array}$ & $\begin{array}{l}\text { No es obligatoria la asistencia } \\
\text { No se entregan trabajos } \\
\text { Examen final: Parte teórica y par- } \\
\text { te práctica }\end{array}$ \\
\hline \multicolumn{3}{|c|}{ Peso en la calificación final } \\
\hline $\begin{array}{l}\text { Proyecto de Aprendizaje Tutorado (PAT): } \\
35 \% \\
\text { Dosier de apuntes y mapas conceptuales: } \\
10 \% \\
\text { Fichas de sesiones prácticas: } 20 \% \\
\text { Recensiones y trabajos individuales (ensa- } \\
\text { yo, tertulias dialógicas...): } 15 \% \\
\text { Examen parcial con coevaluación o eva- } \\
\text { luación entre iguales: } 20 \%\end{array}$ & $\begin{array}{l}\text { PAT: } 30 \% \\
\text { Trabajos prácticos: Hasta un } 20 \% \\
\text { Examen parcial con coevaluación: } \\
50 \%\end{array}$ & $\begin{array}{l}\text { PAT: } 30 \% \\
\text { Examen final con una parte teó- } \\
\text { rica }(50 \%) \text { y una parte práctica } \\
(20 \%) \text { y la presentación de un } \\
\text { informe sobre el PAT que supone } \\
\text { un } 30 \% \text { sobre la calificación final. }\end{array}$ \\
\hline
\end{tabular}

\section{Elaboración propia.}

Durante el desarrollo de la asignatura se llevan a cabo diferentes actividades de aprendizaje y que vamos a explicar brevemente en qué consiste cada una de ellas.

- Proyecto de Aprendizaje Tutorado (PAT): Es un trabajo grupal en el que cada grupo elige un tema de los proporcionados por el profesor y se asignan unos textos para elaborar un marco teórico y plan de sesión acorde al tema. La sesión práctica se lleva a cabo con el resto de compañeros y se expone el marco teórico en 10 minutos. Después de la puesta en práctica, deben realizar un informe en que se reflexione sobre la práctica. Durante todo el proceso se realizan tutorías para corregir los documentos.

- Dosier de apuntes y mapas conceptuales: Durante las sesiones teóricas, los alumnos deben ampliar y reforzar los contenidos del dosier que se les proporciona en la plataforma. En el dosier aparecen pequeñas actividades sobre la teoría: Preguntas, tablas, citas bibliográficas, etc. Además, por cada tema del dosier se elabora un mapa conceptual en el que el alumnado debe demostrar que conoce los contenidos y es capaz de relacionar unos con otros.

- Fichas de sesiones prácticas: Todas las semanas en las que se desarrolla la asignatura, se realizan sesiones prácticas y cada grupo de alumnos debe elaborar una ficha de sesión que debe seguir la siguiente estructura: Narrado de sesión, ventajas, inconvenientes y mejoras de la propuesta, una vivencia personal, un pequeño análisis de las competencias docentes.

- Recensiones y trabajos individuales: Se realizan tres tertulias dialógicas a lo largo de toda la asignatura. Se facilitan dos textos en la plataforma virtual y cada alumno debe leer uno de ello y elegir, al menos, tres párrafos y justificar por qué lo ha hecho. Después, en el aula de manera presencial, se establecen pequeños debates en gran grupo sobre los párrafos que cada compañero ha elegido. Además, en este apartado también realizan un ensayo: Consiste en realizar un pequeño trabajo de 2000 pala- 
bras sobre un tema de educación física de su interés. El ensayo debe tener un resumen, una introducción, un marco teórico, una pequeña propuesta de trabajo, ventajas e inconvenientes, conclusiones y referencias bibliográficas.

- Examen parcial: Se trata de una prueba de conocimientos donde el alumnado colabora en la elaboración de posibles preguntas. También se realiza un proceso de evaluación y corrección entre iguales al terminar el examen, con una plantilla facilitada por el profesorado.

Los instrumentos de obtención de datos son las actas oficiales de calificación de la asignatura y el diario de la profesora. El procedimiento seguido para la recogida de datos de cada alum- no se ha realizado a través de las carpetas colaborativas. Estas carpetas se entregan el día del examen parcial y son una recopilación de todas las actividades de aprendizaje que han realizado a lo largo de la asignatura. Deben estar tanto las primeras entregas como las correcciones tras la retroalimentación de los profesores.

Los profesores revisan cada carpeta colaborativa, tanto la parte individual como la grupal, y en base a los criterios de calificación establecidos al principio de la asignatura se obtiene la calificación final.

\section{Análisis y resultados}

En la tabla 4 presentamos el número de alumnos que han optado por cada vía de aprendizaje y evaluación:

\section{Tabla 4. Número de alumnos en cada vía de aprendizaje y evaluación}

\begin{tabular}{|l|c|c|}
\hline & Porcentaje & $\mathbf{N}^{\circ}$ de alumnos \\
\hline Vía continua & $91.9 \%$ & 34 \\
\hline Vía mixta & $2.7 \%$ & 1 \\
\hline Vía final o no presencial & $5.4 \%$ & 37 \\
\hline Totales & $100 \%$ & 37 \\
\hline
\end{tabular}

La mayoría de los alumnos han optado por la vía de aprendizaje y evaluación continua (91,9\%). En cambio, solo un $2.7 \%$ del alumnado ha optado por la vía mixta por no haber podido entregar todos los trabajos en tiempo y forma adecuados y un $5.4 \%$ de los alumnos han elegido la vía final o no presencial por no poder asistir a las clases. En la tabla 5 presentamos los resultados globales de la asignatura. Es importante destacar el elevado número de alumnos que han superado la asignatura en la primera convocatoria de la asignatura (97.3\%).

Tabla 5. Resultados globales de la asignatura

\begin{tabular}{|l|c|c|}
\hline \multicolumn{1}{|c|}{ Calificación final } & Porcentaje & $\mathbf{N}^{\circ}$ de alumnos \\
\hline Matrícula de honor & $5.4 \%$ & 2 \\
\hline Sobresaliente & $21.62 \%$ & 20 \\
\hline Notable & $54.06 \%$ & 6 \\
\hline Aprobado & $16.22 \%$ & 0 \\
\hline Suspenso & 0 & 1 \\
\hline No presentado & $2.7 \%$ & 37 \\
\hline Totales & $100 \%$ & 8 \\
\hline
\end{tabular}

Las calificaciones finales del alumnado de este curso son bastante buenas, sólo hay un 2.7\% que no ha superado la asignatura porque no se ha presentado al examen. El resto de los alumnos 
han superado la asignatura con calificaciones bastante altas. La nota media de la asignatura es de 7.72 puntos sobre 10. En la tabla 6 se recogen los porcentajes de cada calificación según la vía de aprendizaje y evaluación elegida por los alumnos.

Tabla 6. Porcentajes de cada calificación según la vía de aprendizaje y evaluación elegida por el alumnado

\begin{tabular}{|l|c|c|c|c|c|c|c|}
\hline \multicolumn{1}{|c|}{ Vías } & NP & Suspenso & Aprob. & Notable & Sobres. & Mat. Honor & Totales \\
\hline Continua & - & - & $13.52 \%$ & $51.36 \%$ & $21.62 \%$ & $5.4 \%$ & $91.9 \%$ \\
\hline Mixta & - & - & $2.7 \%$ & - & - & - & $2.7 \%$ \\
\hline Examen & $2.7 \%$ & - & - & $2.7 \%$ & - & - & $5.4 \%$ \\
\hline Totales por calificaciones & $2.7 \%$ & - & $16.22 \%$ & $54.06 \%$ & $21.62 \%$ & $5.4 \%$ & $100 \%$ \\
\hline
\end{tabular}

Teniendo en cuenta estos datos, el rendimiento académico es diferente en función de la vía de evaluación elegida. En primer lugar, todos los alumnos que han seguido la vía continua han superado la asignatura y, además, han obtenido los mejores resultados. El $2.7 \%$ que ha optado por la vía mixta ha obtenido una calificación de aprobado. Con respecto a la vía examen final, encontramos dos casos: Un $2.7 \%$, que ha superado la asignatura con una calificación de notable y un $2.7 \%$ que no se presentó al examen final. Los resultados muestran que las calificaciones más altas se obtienen a través de la vía continua de aprendizaje y evaluación: Notables y sobresalientes en su mayoría. Mientras que la vía final o no presencial solo un $2.7 \%$ consigue aprobar la asignatura con una calificación de notable; una calificación que no suele ser la habitual.

\section{Discusión y conclusiones}

Este trabajo muestra los resultados obtenidos tras la implementación de un sistema de EFyC, combinado con la utilización de metodologías activas, en una asignatura de cuarto curso del Grado de Maestro en Educación Infantil. Por una parte, esta experimentación parece mostrar que la utilización de sistemas de EFyC es la más coherente con el uso de metodologías activas en la FIP.

Se ofrece al alumnado la libre elección de una de las tres vías de aprendizaje y evaluación: Vía continua y EFyC, vía mixta y vía final (examen final). La vía examen final suele ser elegida por los alumnos que no quieren estar implicados de forma continua en su proceso de enseñanzaaprendizaje o que no pueden asistir a clase. En nuestro caso, la mayoría de los alumnos han elegido la vía continua, aunque suponga más trabajo y existan más requisitos (asistencia, entregas de trabajos, etc.). Estos resultados son similares a los encontrados por Julian et al. (2010), MartínezMínguez et al. (2015) y Vallés et al. (2011), que recogen experiencias del alumnado de la FIP que valora de manera positiva la vivencia de sistemas de EFyC durante su formación y están bastante satisfechos con la experiencia, porque generan un mayor aprendizaje, a pesar de que suponga más implicación y más tiempo de trabajo para los alumnos y para el profesorado.

Por otra parte, los resultados muestran un elevado rendimiento académico que, además, parece ser diferente según la vía de aprendizaje y evaluación que elija el alumnado. Similares resultados han sido encontrados también en estudios con otras muestras y contextos (Angelini, 2016; Arribas, 2012; Buscá et al., 2010; Gallardo et al., 2020; López-Pastor et al., 2013), que aseguran que el desarrollo de sistemas de EFyC en la FIP mejora el rendimiento académico del alumnado tanto en España como en Chile. Debido al alto porcentaje de alumnos que han superado la asignatura $(97.3 \%)$, podemos concluir diciendo que parece que el desarrollo de los sistemas de EFyC parecen mejorar el rendimiento académico del alumnado. Estos datos se pueden encontrar también en estudios como los de Brown y Glasner (2003) y Castejón et al. (2011). 
Existen varios trabajos que afirman que el rendimiento académico suele ser mejor siguiendo una vía de evaluación continua, en la que se produce un proceso de mejora del aprendizaje del alumnado gracias a la rápida retroalimentación del profesor (Black \& Wilian, 2003; Boud, 2010; Boud \& Falchikov, 2006; Fraile et al., 2013; López-Pastor, 2008; Romero-Martín, 2014 y 2015).

Los resultados de la vía mixta son ambivalentes: Por un lado, suelen estar las personas que no pueden asistir a todas las clases y, por otro, las personas que no entregan los trabajos o que no cumplen con los criterios mínimos exigidos. En esta situación, los alumnos suelen superar la asignatura $(2.7 \%)$. Estos resultados pueden contrastarse con los recogidos en Castejón et al. (2011), Vallés et al. (2011) y López et al. (2011).

Respecto a la vía de examen final el $2.7 \%$ que sí se ha presentado al examen ha sacado una buena nota (un notable), mientras que el 2.7\% restante no se ha presentado al examen. Esto no suele habitual en esta vía, como puede comprobarse en los estudios de Arribas (2012) y Fraile et al. (2013). Además, en el estudio de Castejón et al. (2011) hace referencia a que por esta vía se suele acumular el trabajo al final del cuatrimestre y repercute en el rendimiento académico, que suele ser bajo.

Como principal limitación de estudio podemos señalar que es una única asignatura y un solo grupo, y que por tanto los resultados no pueden ser generalizables para cualquier caso, pero sí trasferibles a otro contexto. Dado que no se trata de un estudio experimental, cada alumno elige libremente la vía de evaluación y aprendizaje que prefiere. Esto hace que el número de alumnos sea más elevado en la vía de evaluación formativa y continua, lo que podría ser también una limitación del estudio.

Este artículo puede ser de interés para los docentes de la FIP que se inician en las metodologías activas y la EFyC y para los docentes que aplican ya estos sistemas en sus aulas y/o investigan sobre la influencia de la EFyC en el rendimiento académico del alumnado o en las diferentes vías de aprendizaje y evaluación que se puede ofrecer al alumnado en educación superior.

A partir de estos resultados, parece conveniente realizar estudios con muestras más amplias, con diferentes asignaturas que utilizan sistemas de EFyC en la FIP, o bien análisis comparativo del rendimiento académico obtenido en sucesivos cursos y generaciones en esta misma asignatura. Pero, lo más relevante en estos tiempos de confinamiento y obligada enseñanza online en muchos países, debido a la pandemia COVID19, sería analizar en qué medida esta situación de enseñanza no presencial y a distancia ha afectado al desarrollo de los sistemas de EFyC en la FIP.

\section{Referencias bibliográficas}

Angelini-Doffo, M.L. (2016). Estudio sobre la evaluación formativa y compartida en la formación docente en inglés. Revista Actualidades Investigativas en Educación, 16(1), 1-21. http://dx.doi.org/10.15517/aie.v16i1.22614

Arribas, J.M. (2012). El rendimiento académico en función del sistema de evaluación empleado. Relieve, 1(18) 1-15. http://bit.ly/2whMTV5

Biggs, J.B. (2005). Calidad del aprendizaje universitario. Narcea.

Black, P., \& William, D. (2003). In Praise of Educational Research: Formative assessment. British Educational Research Journal, 5(29), 623-637. https://doi.org/10.1080/0141192032000133721

Boud, D., \& Associates (2010). Assessment 2020: Seven propositions for assessment reform in higher education. Australian Learning and Teaching Council.

Boud, D., \& Falchikov, N. (2006). Aligning assessment with long-term learning. Assessment and Evaluation in Higher Education, 31, 4, 399-413. https://doi.org/10.1080/02602930600679050

Brown, S., \& Glasner, A. (2003). Evaluar en la Universidad. Problemas y nuevos enfoques. Narcea.

Buscá, F., Pintor, P., Martínez, L., \& Peire, T. (2010). Sistemas y procedimientos de Evaluación 
Formativa en docencia universitaria: Resultados de 34 casos aplicados durante el curso académico 2007-2008. Estudios Sobre Educación, 18, 255-276. http://bit.ly/2QqXJPv

Cañadas, L., Santos-Pastor, M.L., \& Castejón F.J. (2018). Desarrollo de competencias docentes en la Formación Inicial del Profesorado de Educación Física. Relación con los instrumentos de evaluación. Estudios Pedagógicos, 44(2), 111-126. http://dx.doi.org/10.4067/S0718-07052018000200111

Carter-Thuillier, B. (2015). Evaluación para el aprendizaje: Innovación, Democracia y Transformación en Educación Física. En M. Cresp, J. Serra-Olivares, B. Carter-Thuillier \& R. García (Eds.), Evaluación para el aprendizaje: Educación Física e Innovación en procesos formativos (pp. 24-49). Lambert-EAE.

Castejón Oliva, F.J., López-Pastor, V.M., Julián Clemente, J.A., \& Zaragoza Casterad, J. (2011). Evaluación formativa y rendimiento académico en la formación inicial del profesorado de Educación Física. Revista Internacional de Medicina y Ciencias de la Actividad Física y el Deporte, 11(42), 328346. http://bit.ly/2WoLw1C

Dochy, F., Segers, M., \& Dierick, S. (2002). Nuevas vías de aprendizaje y enseñanza y sus Consecuencias: Una nueva era de evaluación. Revista de la Red Estatal de Docencia Universitaria, 2(2), 13-29. http://bit.ly/3a0OEEX

Falchikov, N. (2005). Improving Assessment Through Student Involvement. Practical solutions for aiding learning in higher and further education. Routledge.

Fraile-Aranda, A., López-Pastor, V.M., Castejón-Oliva, F.J., \& Romero-Martín, R. (2013). La evaluación formativa en docencia universitaria y el rendimiento académico del alumnado. Revista Aula Abierta, 41(2), 23-34. http://bit.ly/2WncDtJ

Gallardo, F., Carter, B., \& López-Pastor, V. M. (2019a). Evaluación formativa y compartida en la universidad chilena: Resultados tras cuatro años de implementación. Revista Iberoamericana de Evaluación Educativa, 12(1), 139-155. https://doi.org/10.15366/riee2019.12.1.008
Gallardo-Fuentes, F., López-Pastor, V.M., MartínezAngulo, V., \& Carter-Thuillier, B. (2019b). Evaluación formativa en educación física y atención a la diversidad. Percepción del alumnado de educación física. Revista Internacional de Investigación en Educación, 12 (25), 169-186.

https://doi.org/10.11144/Javeriana.m12-25.efef

Gallardo-Fuentes, F., López-Pastor, V.M., \& CarterThuillier, B. (2017). ¿Hay evaluación formativa y compartida en la formación inicial del profesorado en Chile? Percepción de alumnado, profesores y egresados de una universidad. Psycology, Society \& Education, 9(2), 227-238.

https://doi.org/10.25115/psye.v9i2.699

Gallardo-Fuentes, F., López-Pastor, V.M., \& CarterTuhillier, B. (2018). Efectos de la aplicación de un sistema de evaluación formativa en la autopercepción de competencias adquiridas en formación inicial del profesorado. Revista Estudios Pedagógicos, 44(2), 55-77. https://bit.ly/3dCblkz

Gallardo-Fuentes, F., López-Pastor, V., \& CarterThuillier, B. (2020). Ventajas e inconvenientes de la evaluación formativa, y su influencia en la autopercepción de competencias en alumnado de Formación Inicial del Profesorado en Educación Física. Retos, Nuevas tendencias en Educación Física, Deporte y Recreación, 38, 417-424. https://bit.ly/30c5YEU

Hamodi, C., López-Pastor, V. M., \& López-Pastor, A.T. (2017). If I experience formative assessment whilst at University will I put it into practice later as a teacher? Formative and shared assessment in Initial Teacher Education. European Journal of Teacher Education, 40(2), 171-190. https://doi.org/10.1080/02619768.2017.1281909

Herranz, M. (2013). Desarrollo de procesos de autoevaluación y evaluación compartida en la etapa de Educación Primaria. Un estudio de casos en el área Educación Física [Tesis Doctoral, Universidad de Valladolid, Segovia]. http:// bit.ly/2Uf4a9v

Ibarra, M.S., Rodríguez, G., \& Gómez, M. A. (2012). La evaluación entre iguales: Beneficios y estrategias para su práctica en la universidad. Revista de Educación, 359, 206-231. 
https://doi.org/10.4438/1998-592X-RE-2011-359-092.

Julián, J.A., Zaragoza, J., Castejón, F.J., \& LópezPastor, V.M. (2010). Carga de trabajo en diferentes asignaturas que experimentan el sistema ECTS. Revista Internacional de Medicina y Ciencias de la Actividad Física y el Deporte, 10(38), 218-233. http://bit.ly/2vwxmAl

López Pastor, V.M. (2008). Desarrollando sistemas de evaluación formativa y compartida en la docencia universitaria. Análisis de resultados de su puesta en práctica en la formación inicial del profesorado. European Journal of Teacher Education, 31(3), 293-311. https://doi.org/10.1080/02619760802208452

López-Pastor, V.M., Archilla, M., \& Real, F. (2016). Evaluación formativa, calificación y carpetas colaborativas: Una práctica de éxito en educación superior. In C. Hamodi Galán (Coord.), Formar mediante la evaluación en la Universidad. Propuestas prácticas útiles para docentes (pp. 67-82). Ediciones Universidad de Valladolid

López-Pastor, V.M., Manrique, J.C., \& Vallés, C. (2011). Evaluación y calificación en los nuevos estudios de Grado. Especial incidencia en la Formación Inicial del Profesorado. Revista Electrónica Interuniversitaria de Formación del Profesorado, 39(14.4), 1-20. http://bit.ly/33E0pih

López-Pastor, V.M., Molina, M., Pascual, C., \& Manrique, J.C. (2020). La importancia de utilizar la Evaluación Formativa y Compartida en la formación inicial del profesorado de Educación Física: Los Proyectos de Aprendizaje Tutorado como ejemplo de buena práctica. Retos, Nuevas tendencias en Educación Física, Deporte y Recreación, 37, 680-687. http://bit.ly/2wlylUi

López-Pastor, V.M., \& Pérez-Pueyo, A. (Coords.) (2017). Evaluación formativa y compartida en Educación: Experiencias de éxito en todas las etapas educativas. Universidad de León. http://bit.ly/2J2BRFP

López-Pastor, V., Pintor, P., Muros, B., \& Webb, G. (2013). Formative assessment strategies and their effect on student performance and on student and tutor workload: The results of research projects undertaken in preparation for greater convergence of universities in
Spain within the European Higher Education Area (EHEA). Journal of Further and Higher Education, 37, 163-180.

https://doi.org/10.1080/0309877X.2011.644780

Martínez Carazo, P.C. (2006). El método de estudio de caso. Estrategia metodológica de la investigación científica. Pensamiento y gestión, 20, 165-193. http://bit.ly/2WndjPN

Martínez-Mínguez, L., Vallés-Rapp, C., \& RomeroMínguez, M.R. (2015). Estudiantes universitarios: Ventajas e inconvenientes de la evaluación formativa. Revista d'Innovació educativa, (14), 59-70. https://doi.org/10.7203/attic.14.4217

Montero, I., \& León, O.G. (2005). Sistema de clasificación del método en los informes de investigación en Psicología. International Journal of Clinical and Health Psychology, 5(1), 115-127. https://bit.ly/3gUCtgA

Moreno Ruiz, J.A., Candela Martín, A., \& Bañuelos Lagunes, P. (2019). Evaluaciones formativas en el aula: Análisis discursivo de la actividad de retroalimentación en la práctica supervisada de psicólogos educativos en formación. Revista Iberoamericana de Evaluación Educativa, 12(1), 121-137. https://doi.org/10.15366/riee2019.12.1.007

Nicol, D., \& MacFarlane-Dick, D. (2006). Formative assessment and self-regulated learning: A model and seven principles of good feedback practice. Studies in Higher Education, 31(2), 199-218. https://doi.org/10.1080/03075070600572090

Romero-Martín, M.R., Asún, S., \& Chivite, M.T. (2016). La autoevaluación en expresión corporal en formación inicial del profesorado de educación física: Un ejemplo de buena práctica. Retos. Nuevas tendencias en Educación Física, Deportes y Recreación, 29, 236-241. http://bit.ly/38ZKsE1

Romero-Martín, R., Fraile-Aranda, A., López-Pastor, V.M., \& Castejón-Oliva, F.J. (2014). Relación entre sistemas de evaluación formativa, rendimiento académico y carga de trabajo del profesor y del alumno en la docencia universitaria. Revista Infancia y Aprendizaje, 37(1), 16-32.

http://dx.doi.org/10.1080/02103702.2014.918818 
El rendimiento académico y la evaluación formativa y compartida en formación del profesorado

Romero-Martín, R., Castejón-Oliva, F.J., \& LópezPastor, V.M. (2015). Divergencias del alumnado y del profesorado universitario sobre las dificultades para aplicar la evaluación formativa. RELIEVE, 21(1), 1-16. https://doi.org/10.7203/relieve.21.1.5169
Vallés, C., Ureña, N., \& Ruiz, E. (2011). La evaluación Formativa en Docencia Universitaria. Resultados globales de 41 estudios de caso en su primer año de desarrollo. Revista de Docencia Universitaria, 9(1), 135-158. http://bit.ly/2Uhg8PJ 